\title{
SOME NONLINEAR PROBLEMS \\ IN THE \\ MANIPULATION OF BEAMS
}

\author{
Andrew M. Sessler* \\ Lawrence Berkeley Laboratory \\ University of California \\ Berkeley, CA 94720
}

October 1,1990

\begin{abstract}
An overview is given of nonlinear prcblems that arise in the manipulation of beams. Beams can be made of material particles or photons, can be intense or dilute, can be energetic or not, and they can be propagating in vacuum or in a medium. The nonlinear aspects of the motion are different in each case, and this diversity of behavior is categorized. Many examples are given, which serves to illustrate the categorization and, furthermore, display the richness of behavior encountered in the physics of beams.
\end{abstract}

\section{INTRODUCTION}

Most physicists look upon beam physicists as engineers. True, sophisticated engineers, but nevertheless engineers. This, because they work in an area where the basic understanding has long since been obtained and the subject of the trade is the practical application, with due regard for its many aspects, of this knowledge.

It is certainly true that in the construction of beam-handling-devices full attention must be paid to proper, and careful, engineering. And attention must also be given to the proper execution of concept. History is replete with the stories of machines, or components, that "didn't work" for various "trivial" reasons. Let me give an example: even this year, and at a very sophisticated laboratory, namely CERN, it was discovered that an extremely thin nickel layer that was employed to bond a lead

* Work supported by the Office of Energy Research, US Department of Energy, under Contract No. DE-AC03-76SF00098. 
shield to an aluminium vacuum chamber had excessive, and undesirable, magnetic properties which limits the performance of the new, $27 \mathrm{~km}$, collider called LEP.

It is very true that beam physicists have to be aware of engineering, perhaps to a greater degree than most physicists, but with "big science" pervading much of physics, other physicists have also to be concerned with engineering. Think for a moment of space science (with its satellites and remote sensing devices), or radio and optical astronomy (with its large telescopes). And, taking astronomy as an example, it isn't difficult to think of cases where the engineering failed: either in the collapse of a ground based radio telescope or in the aberrations of an orbiting lens.

It is not true that complicated phenomena (nonlinear) are characterized as "physics" and simple phenomena (linear) as "engineering". Engineering often deals with very complicated, nonlinear, phenomena such as turbulent flow with viscous boundary layers (and engineers understand this well enough to design commercial jets) or the strength of materials (which knowledge is used ubiquitously). Rather, the distinction between physics and engineering is based upon the degree of understanding: "handy formulas" valid for the usual (albeit very complicated case) versus novel, and not understood, situations.

Beam physicists really are physicists. There are a great many areas in the physics of beams where the subject is not engineering, i.e., where the fundamental phenomena is not well-understood, or at least it was not well-understood when it was first encountered. I would like in this review to cover some of these topics.

In addition, and most appropriate for this Workshop, they are all nonlinear, for linear phenomena are well-understood. In fact they are so well-understood that if it weren't for nonlinear phenomena accelerators and partiws handling machines would work perfectly; i.e., accelerators would create arbitrarily large currents and colliders would have arbitrarily large luminosities. Beam physicists work upon the subjects of why they don't work perfectly, and how to improve their performance.

In the next section (Section II) we shall categorize the various phenomena, and then in the following section (Section III) give a good number of examples. Some of these examples will be of rather well-known phenomiena. Jut some will be of topics on the frontiers of present research. 


\section{CATEGORIZATION}

In Table 1 is indicated a convenient manner in which to categorize nonlinear phenomena encountered in the study of particle and photon beams. The Table is not complete, but it covers all of the nonlinear phenomena currently under study by beam physicists. I would like to discuss, now, the various categories.

Table 1. A categorization of nonlinear behavior encountered in the study of particle and photon beams.

Dilute Single Beam in

Vacuum

Intense Single Beam

Two Intense Beams

Particle Beam in Media

Particle Beam and

$\underline{\text { Radiation }}$

Photon Beam in a Media
Nonlinear Aperture Limit

Longitudinal Motion

Interaction with an Intense Beam

Space Charge Effects

Linear Coherent Instability, but with

Nonlinearities Creating a Threshold

Nonlinear Coherent Instability

Radiation, Particle Creation,
Disruption, Coherent Motion

Coherent Light Gener:tion by

Nonlinear Particle Motion

Light Behavior in the Electron Beam
Frequency and Wavelength Changes when the Media is Spatially and Temporarily Changing

Firstly, a beam handling device is designed so that it "works" for a single particle; i.e., many-particle, collective, effects are ignored. Thus the first nonlinear problems that one encounters are those associated with a dilute beam. The dilute beam is, of course, a particle beam and not a photon beam, since the linearity of Maxwell's equations imply no nonlinear phenomena for light beams. (Strong light beams moving in a media can disturb the media, and thus can experience nonlinear effects, and this is included in the very last category.)

Nonlinear behavior of a dilute beam is a much-studied subject. There are deep questions associated with even this relatively simple case of nonlinear single-particle Hamiltonian motion. For a beam physicist a most important aspect is "Dynamic 
Aperture"; i.e., what is the available space for accelerating, or holding, particles in an accelerator? Longitudinal manipulation is usually highly nonlinear motion, and understanding of this aspect has been instrumental in advancing the capability of beam physicists.

For two beams interacting with each other, which subject is of great importance, for almost all high energy machines are colliders, the approximation is often made of "weak-strong"; i.e., not having one of the beams (the strong beam) influenced by the other (the weak beam). Even with this approximation the behavior is extremely complicated.

After designing a beam handling device taking into account single particle effects, beam physicists turn, secondly, to phenomena associated with intense beams. This is where the limits on accelerated, or stored; currents are encountered. Firstly, one is concerned with equilibrium flows, i.e., the design of cathodes and ion sources and various "space charge limits". Secondly, one is concerned with collective dynamic effects. Much of the analysis is done in linear approximation. The collective motion usually is "bad", and it is prevented by Landau damping, which generally is created by nonlinear phenomena.

Two intense beams have behavior which can be even more complicated than that disclosed in the "strong-weak" approximation. For example there can be the nonlinear quantum phenomena of the creation of particles. (This effect is not always 2 "good" one; in high energy linear colliders electron pairs can be copiously produced, and the detectors are swamped with well-understood QED phenomena, while strong, or weak, interaction phenornena, which is what one is really interested in, go undetected.) Leaving aside quantum phenomena, there are highly nonlinear beam-beam phenomena, which are of great interest, such as the disruptive effect of beams upon each other.

Once a particle leam is sent into a media all sorts of things can occur. Lots of study has been made of the nonlinear motion, created in the media, in most cases a plasma, by the beam. The phenomena are, surely, important, but are only barely studied, in contrast with those phenomena previously mentioned.

Particle beams interacting with radiation are the basis for many coherent wave generation devices: klystrons, magnetrons, free-electron lasers, backward wave 
oscillators, etc. The particle motion is almost always nonlinear. The radiation is affected by the particles, and so the radiation also behaves nonlinearly.

The final category, in Table 1, is on the cutting edge of beam physics pheromena, and appears to hold the promise of making some very interesting devices.

\section{EXAMPLES}

\section{III.1 Dynamic Aperture}

It was in the 1950's that physicists first became interested in the nonlinear nature of single particle motion in accelerators. Strong focusing, which was really the idea, and the methods of analysis, of diverse transverse focusing elements, and then its immediate generalization to fixed field alternating gradient accelerators (FFAG), required that accelerator physicists be concerned about the effect of strong nonlinearities in the transverse motion of particles. Because FFAG allowed the storage of particles, for the first time physicists saw the way to realizing a collider. This, however, required a much deeper understanding of longitudinal behavior, also very nonlinear, than had ever been achieved (or needed) prior to that time; in the next section we comment a bit about the longitudinal motion.

At first, the question was raised as to whether nonlinear motion was intrinsically unstable. (Remember, this was even before the KAM Theorem!) Colliders require stability for the order of $10^{11}$ turns, which meant that little comfort could be obtained form the observed stability of the solar system for at least $10^{9}$ years.

Some analytic. work was done to disclose the nature, in some simple cases, of stable and unstable fixed points. It was soon realized that small amplitude motion, if not stable for infinite times, was at least quite stable compared to that of large amplitude motion. Then, in some very early studies, we taxed the computers of the day, used Hamiltonian transformations (we realiz".d it was important to have them Hamiltonian) for a section of circumference; concatenated and iterated the transformations and were able to study behavior, in some realistic machines, for 50,000 turns. It wasn't until 15 years later that experimental demonstration of stability was achieved (and hence a great worry was removed, when the very large, and expensive, machine, the ISR, "worked"). 
In the passing years it has become clear that machines cannot be built having their "natural" chromaticity (variation of tune with energy), but the chromaticity must be controlled. This is most easily done by introducing sextapoles; i.e., nonlinear elements into the lattice. The result has been to introduce into linear machines elemunts that make them nonlinear (as were the old FFAG machines). Thus interest has continued in studying the effect of nonlinearities.

Not only must sextapoles be put into high energy physics colliders, but machines built as synchrotron radiation sources profit from having special inserts, often highly nonlinear, such as wigglers. Again, there is great interest in studying the effect of nonlinearities.

Although analytic work is done, for example using Lie Algebra techniques, almost all work is done by computer simulation, i.e., single particle tracking. 1,2 The codes for doing this are very extensive and, as one can imagine, ernploy a great deal of super computer time. They include linear elements, nonlinear effects, magnet errors (in excitation, in placement, and intrinsically nonlinear). One is interested in determining the dynamic aperture, which is the area within a particle that will exhibit stable betatron and synchrotron motion. Nevertheless, as one can see readily from Fig. 1, considerable extrapolation is needed to go from the computer results (which in this case required 7 hours of CPU time) to the desired storage time of 10 hours. ${ }^{3}$ In the case shown, a simple linear extrapolation (on a semi-log plot) would imply a $40 \%$ reduction in dynamic aperture as one goes from ten thousand turns to a billion turns. Thus, although the extrapolation needs to be large, the numerical studies are extremely valuable, and the subject is pursued extensively.

\section{2 Longitudinal Motion}

An understanding of longitudinal motion can be obtained rather quickly from an examination of the motion of a single particle under the influence of a single rf cavity. 4 

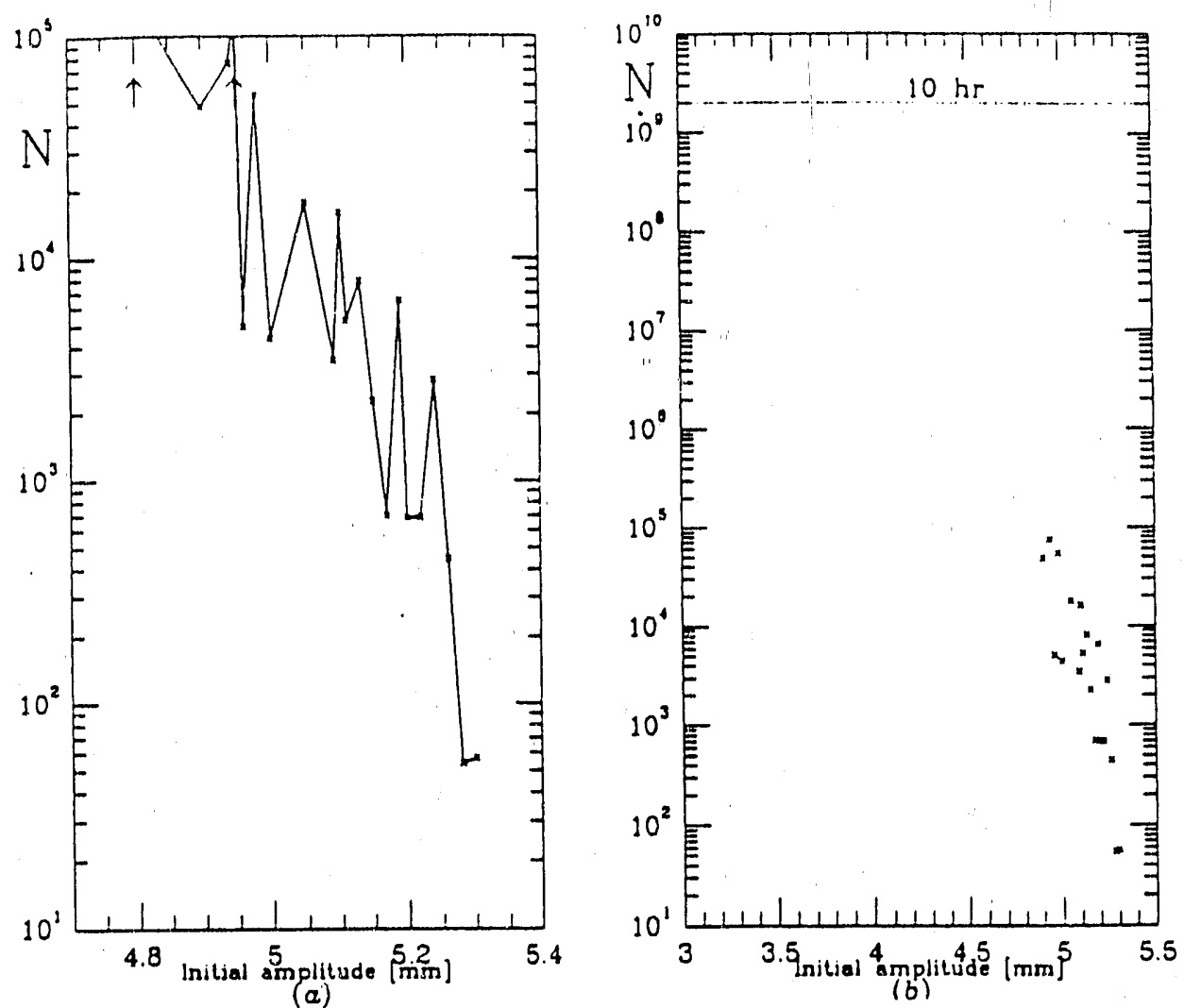

Fig. 1 The number of turns a particle survives as a function of horizontal displacement $\mathrm{x}_{0}$ (all other coordinates zero) (a). The same results scaled to Fermilab Collider operation (b). (Figure from Ref. 3 ).

The accelerator is completely characterized by a single function of particle energy; namely, the longitudinal dispersion in revolution frequency, usually called $\eta$, such that

$$
\frac{\mathrm{df}}{\mathrm{dp}}=-\frac{\mathrm{fn}}{\mathrm{p}} \text {, }
$$

where $f$ is the longitudinal frequency of a particle of momentum $p$ and $\eta$ is a given function of $p$.

Taking the rf cavity voltage as

$$
V=V_{0} \sin 2 \pi f_{r f} t
$$

where $f_{r f} h \approx f$, and $h$ is the harmoniz number, one obtains an equation for the phase, $\varphi$, of a particle with respect to the rf wave. That equation is simply: 


$$
\frac{\mathrm{d}^{2} \varphi}{\mathrm{dt}^{2}}+\left[2 \pi \text { he } \mathrm{V}_{0} \mathrm{f} \frac{\mathrm{df}}{\mathrm{dE}}\right] \sin \varphi=2 \pi\left(\frac{\mathrm{df}}{\mathrm{dt}}\right)
$$

which is a driven pendulum equation.

It is possible to form a Hamiltonian for the system and hence employ the powerful methods, and theorems, of classical dynamics. ${ }^{4}$ It is easy to show that the quantity

$$
w=\int^{E} \frac{d E^{\prime}}{f\left(E^{\prime}\right)}
$$

is canonically conjugate to phase $\varphi$.

The subject of adiabatic capture of particles, phase displacement of particles, etc., namely an understanding of nonlinear longitudinal motion, has developed through the years and now is an essential tool of beam physicists. 5

Not only has this been of practical importance in designing capture and extraction systems, but having these concepts deeply ingrained into beam physicists allows them to quickly analyze new devices. This was particularly true for the free electron laser and we shall touch upon this in Section III.7.

\section{III.3 Beam-Beam Limit}

The effect of an intense beam on the other beam, in a collider, is one of the most important phenomena in nonlinear beam physics. The effect limits the performance of almost all colliders and yet is not fully understood.

Consider, so as to grasp the essentials of the subject, the simple case of a single particle interacting with an intense beam by means of a head-on collision. 6 The particle will be kicked and its transverse momentum, $\mathrm{p}_{y}$, will be changed by

$$
\Delta p_{y}=\int F \cdot d t=\left(e E_{y}\right)\left(\frac{\sigma_{z}}{2 c}\right) 2 \sqrt{2 \pi}
$$


where $\mathrm{eE}$ is the electric force, the factor of two is because the magnetic force is equal to the electric force, and $\sigma_{z} / 2 \mathrm{c}$ is the time of interaction for a bunch of length $\sigma_{z}$.

In linear approximation a bunch of $\mathrm{N}$ particles, and dimensions $\sigma_{\mathrm{x}} \times \sigma_{\mathrm{y}} \times \sigma_{\mathrm{z}}$, produces a deflecting field

$$
E_{y}=\frac{N e y}{\sigma_{x} \sigma_{y} \sigma_{z}} \frac{2}{\sqrt{2 \pi}}
$$

Thus a particle experiences a deflection

$$
\Delta\left(\frac{\mathrm{dy}}{\mathrm{ds}}\right)=\frac{\Delta \mathrm{p}_{\mathrm{y}}}{\mathrm{p}}=\frac{2 \mathrm{~N} \mathrm{r}_{\mathrm{o}} \mathrm{y}}{\sigma_{\mathrm{x}} \sigma_{\mathrm{y}} \gamma}
$$

where $r_{0}$ is the classical electron radius and $\gamma$ is the relativistic factor. In circular machines one introduces the tune parameter $v_{y}$ which is the number of betatron (transverse) oscillations in one turn. Defining the "local betatron wavelength", $\lambda$, in terms of a parameter $\beta, \beta=2 \pi / \lambda$, we obtain

$$
\begin{aligned}
& \Delta v_{y}=\frac{\Delta \lambda}{2 \lambda}=\frac{1}{2 \lambda} \Delta\left(\frac{d y}{d s}\right) \frac{1}{y} \\
& \Delta v_{y^{\prime}}=\frac{N r_{o} \beta}{2 \pi \sigma_{x} \sigma_{y} \gamma}
\end{aligned}
$$

What has been observed for decades, and on many different colliders, is that $\Delta v_{y}$ is limited to unreasonably small values. ${ }^{7,8}$ For a purely linear effect (which could be easily corrected by quadrupoles somewhere else on the circumference of the collider) $\Delta v$ would be limited to $\approx 0.2$. In fact $\Delta v_{y} \leq 0.02$ to 0.06 .

The non-linear effect has been ex:ensively studied both theoretically and experimentally. 7,8 . .ote that even the nonlinear effects are characterized by $\Delta v_{y}$ (there is no other parameter in the problem). Thus a "linear parameter", $\Delta v$, is extensively discussed, but everyone knows the effect is highly (by an order of magnitude) nonlinear.

In some machines $\Delta v_{y} \approx 0.06$, at other times, or in other machines, it is very small. The dependence on operating conditions is severe (showing that one must include more aspects of the collider than we did above). The dependence upon the number of crossing points, upon 
electrons vs. protons (i.e., damped vs. Hamiltonian systems), and upon various other things, are all very much a current subject of research.

\section{III.4 Beam Break Up}

In a long linear machine (but quite analogous behavior also occurs in circular machines), due to jitter or misalignment, a slice of the particle beam will move off the center line, or beam line, of the accelerating structure. The resulting dipole component of perpendicular current will excite the structure. A slice of beam, to the rear of the first slice, will experience a $\vee \times \mathrm{B}$ force which changes its betatron amplitude and phase and this slice will now, in the next structure, have even a larger effect on the following part of the beam. An instability, beam break up (BEU), occurs. ${ }^{9}$

The phenomena is described by the equation

$$
\left[\frac{\partial}{\partial z} \gamma_{i}(\tau, z) \frac{\partial}{\partial z}+\gamma_{i}(\tau, z) \mathrm{k}_{\beta i}^{2}(\tau, z)\right] \xi_{i}(\tau, z)=\int_{0}^{s} \mathrm{~d} \tau^{\prime} \frac{\mathrm{I}\left(\tau^{\prime}\right)}{\mathrm{IA}} \mathrm{W}\left(\tau \cdots \tau^{\prime}, \mathrm{z}\right)\left\langle\xi\left(\tau^{\prime}, \mathrm{z}\right)\right\rangle,
$$

where the $i^{\text {th }}$ particle has transverse displacement $\xi_{i}(\tau, z)$ and energy $\gamma_{i}(\tau, z)$. The longitudinal coordinate is $z$ and $\tau=t-z / v_{z}$ indexes beam slices, with $\tau=0$ being the beam head. The beam current is $\mathrm{I}(\tau)$ and $\mathrm{I}_{\mathrm{A}} \equiv \mathrm{mc}^{3} / \mathrm{e}$ is the Alfven current. The restoring force of the linac is characterized by $k_{\beta}^{2}, \lambda_{\beta}=2 \pi / k_{\beta}$, and the effect of one part of the beam on another is completely included in the wake potential, or Green's function, $\mathrm{W}(\tau) .^{9}$

For our purposes, here, we can specialize to the wake created by a smooth resistive wall. (Even in this case there will be BBU.) The wake potential is

$$
W(\tau)=\frac{4}{\sqrt{\pi}} \frac{1}{\tau_{D}^{1 / 2} b^{2} \tau^{1 / 2}}
$$

where $b$ is the radius of a cylindrical pipe through which the beam moves, and

$$
\tau_{D}=\frac{4 \pi \sigma b^{2}}{c^{2}}
$$

where $\sigma$ is the conductivity of the pipe walls. 
Asymptotically, the beam centroid $\left\langle\xi_{\mathrm{i}}\right\rangle$ increases axponentially with distance, $z$, down the linac as

$$
\exp \left(z / L_{g}\right)^{2 / 3}
$$

where the characteristic length, $\mathrm{L}_{\mathrm{g}}$, is given by 10

$$
L_{g}=\frac{2^{7 / 2}}{3^{3 / 2}} \pi^{3 / 2} \frac{I_{A}}{I} \frac{\sigma^{1 / 2} \gamma^{3}}{\tau^{1 / 2} \lambda_{\beta} c} \text {. }
$$

So far, the phenomena has been described in purely linear terms, and this is characteristic of the analysis of most collective instabilities. However the instability is prevented by Landau damping; that is, by a variation in frequencies (or $\lambda_{\beta}$ ) of the particles in a slice which, almost always, is a nonlinear effect. Thus, since beam physicists are in the business of preventing instabilities, they are very much interested in the nonlinear nature of linear accelerators. Another damping mechanism is possible in the case presented, namely, variation of $\gamma$ with $\tau$. This is called BNS damping. ${ }^{11}$ In fact, this damping is even more effective than Landau damping in many cases. In Fig. 2 is shown growth of the instability in the absence of damping, and in Fig. 3 the effect of BNS damping.

\section{III.5 Disruption}

When two beams are brought into collision in a linear collider, so as to produce interesting high energy physics events (large angle scatterings or production of other particles), it is desirable to have the beams very intense so that the rate of interesting events is adequately large. In this situation the bearns have a very strong and nonlinear effect upon each other. ${ }^{12}$

An estimate of this disruption can be obtained by considering a particle at a typical vertical distance, $\sigma_{\mathrm{y}}$, intersecting the opposite bunch of $\mathrm{N}$ electrons distributed 


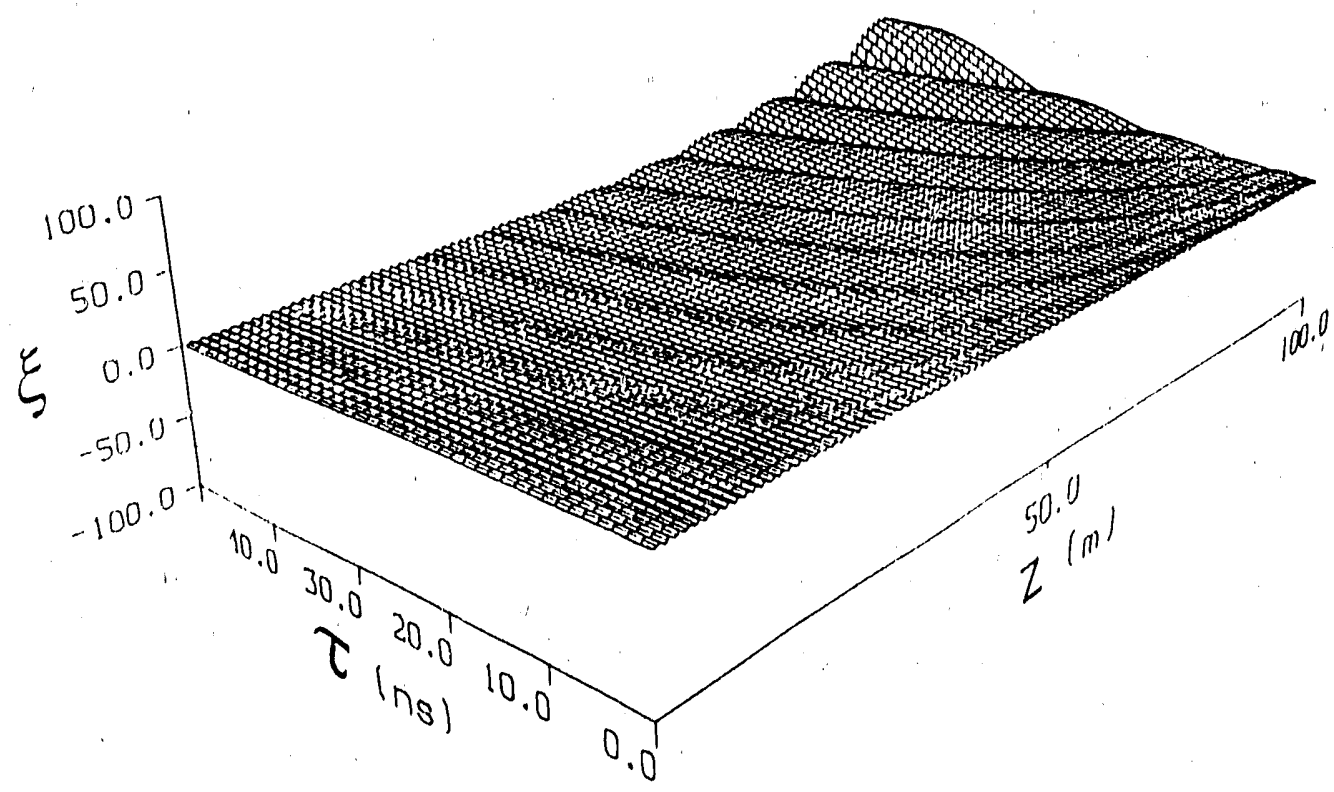

Fig. 2 Resistive wall growth for typical parameters $\left(I=3 \mathrm{kA}, \gamma=2, \lambda_{\beta}=1\right.$ meter, $\left.\sigma=10^{17} \mathrm{sec}^{-1}, \mathrm{~b}=1.0 \mathrm{~cm}\right)$.

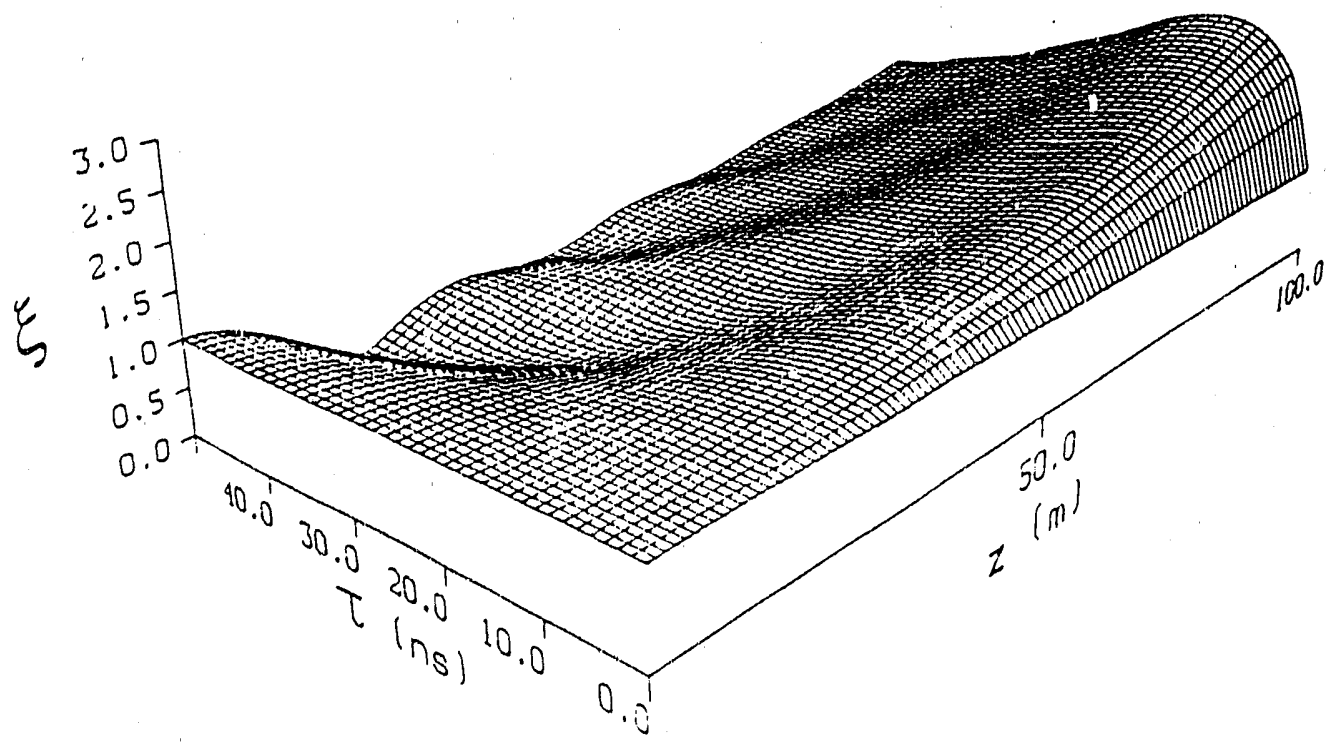

Fig. 3.The damping effect of BNS with $\frac{\Delta \gamma}{\gamma}=-4.0 \%$. (Parameters tho same as in Fig. 2.) 
over a length $\sigma_{\mathrm{z}}$. Because the electric force is equal to the magnetic force, we have for a round beam

$$
\Delta \mathrm{p}_{\mathrm{y}}=\mathrm{F} \cdot \mathrm{t}=2\left(\frac{\mathrm{e}^{2} \mathrm{~N}}{\sigma_{\mathrm{y}}}\right)\left(\frac{\sigma_{\mathrm{z}}}{\mathrm{c}}\right)
$$

Defining the disruption parameter, $\mathrm{D}$, by

$$
D=\frac{\Delta p_{y}}{p} \text {, }
$$

we find:

$$
D=\frac{2 r_{0} N \sigma_{z}}{\sigma_{y}^{2} \gamma}
$$

A careful analysis for Gaussian beams of elliptical cross section yields

$$
D=\frac{2 r_{0} N \sigma_{z}}{\sigma_{y}^{2} \gamma(1+R)}
$$

where $\mathrm{R}=\sigma_{\mathrm{x}} / \sigma_{\mathrm{y}}$, in agreement with the simple derivation given above.

Because in a linear collider the beams are not re-circulated, there is not the restriction on the nonlinear effect of one beam upon the other, analogous to the very severe restriction imposed by $\Delta v_{y} \leq 0.02$ (as discussed in section III.3). The severity of the collision is limited by the coherent production of photons and electron pairs, but can, nevertheless, be very large.

Careful numerical studies of the phenomena have been made. ${ }^{12,13}$ In particular, it has heen shown that the beams self pinch, thus increasing the luminosity (a "good" effect), but create large transverse momenta which causes splattering of particles into the detector (a "bad" effect). 


\section{III.6 Plasma Lens}

When a beam is sent in to a plasma there are two possibilities. Either, where $n_{p}$ is the plasma density and $n_{b}$ is the beam iensity, $n_{p}>n_{b}$ (overdense case) or $n_{p}<$ rib (underdense case). The overdense case is easier to treat (the beam is only a small perturbation to the plasma and linear theory may be employed). ${ }^{14}$ It is the underdense case to which I want to call your attention. 15

In the underdense case, and for an incidunt electron beam, the plasma electrons are pushed to a large radius (the channel radius) by the bearn and the ions (which only move slowly) provide focusing for the electron beam. This focusing force can be very large; i.e.,

$$
F_{y}=-2 \pi e^{2} n_{p} y
$$

or, equivalently,

$$
\frac{F_{y}}{y}=\left[3 \frac{\text { Gigagauss }}{\mathrm{cm}}\right]\left[\frac{\mathrm{n}_{\mathrm{p}}}{10^{18} \mathrm{~cm}^{-3}}\right] .
$$

This large focusing force allows one to construct a plasma lens. For example, with $\mathrm{n}_{\mathrm{p}}=10^{18} \mathrm{~cm}^{-3}$ and a length of only $1 \mathrm{~cm}$, the focal length is only $10 \mathrm{~cm}$ for $50 \mathrm{GeV}$ electrons.

Of course there are many problems which must be addressed, and many of them have already been attacked at least in a preliminary manner, such as the motion of the ions, the very non-linear motion of the plasma electrons, ion channel instabilities, etc.

In fact, ion channels are interesting, not only as a discrete lens element, but also for continuous focusing. ${ }^{15}$ They also are of interest as radiation generators. ${ }^{16}$

Finally, I note that if the beam is made of positrons the situation is very different from that described above, very nonlinear, but that the plasma still provides some significant focusing. 


\section{III.7 High-Power Free-Electron Lasers}

In order for a free electron laser (FEL) to produce very high power it is necessary to taper the wiggler. 17 That is, an FEL operates when the resonance condition

$$
\lambda_{s}=\frac{\lambda_{w}}{2 \gamma^{2}}\left(1+a_{w}^{2}\right)
$$

is observed throughout the wiggler. In this condition $\lambda_{\mathrm{s}}$ is the wavelength of the signal field, $\lambda_{w}$ is the wavelength of the wiggler and $a_{w}$ is expressed in terms of the peak magnetic field, $B_{w}$, by

$$
a_{w}=\frac{e B_{w} \lambda_{w}}{2 \pi \sqrt{2} \mathrm{mc}^{2}} .
$$

As an electron moves through the wiggler it loses energy and hence $\gamma$ decreases. In order to maintain the resonance condition $\lambda_{w}$ must be changed. Usually $a_{w}$ is changed since $\lambda_{\mathrm{w}}$ is not easily modified.

A wiggler must, also, capture a large fraction of the injected electrons in order to produce large FEL power. This is a highly nonlinear process that is not well understood. In Fig. 4 we see a particle simulation of capture and extraction in a particular FEL. Also shown are the stable phase areas given by the theory described in Section III.2. The theory of an FEL can be easily employed when the stable region grows slowly (adiabatically), but usually, in practice, that is not the case.

A rather complete $1 D$ theory of the linear growth of the FEL instability has been presented. ${ }^{18}$ However, there are important matters, such as the stabilization level of the signal field, or the effect of tapering, which are beyond the linear theory. Of course these nonlinear effects can be estimated, and numerical simulation is widely employed to evaluate many things, but much analytic work remains to be done. In particular, such a nonlinear theory would be most useful for analyzing novel FEL configurations. 

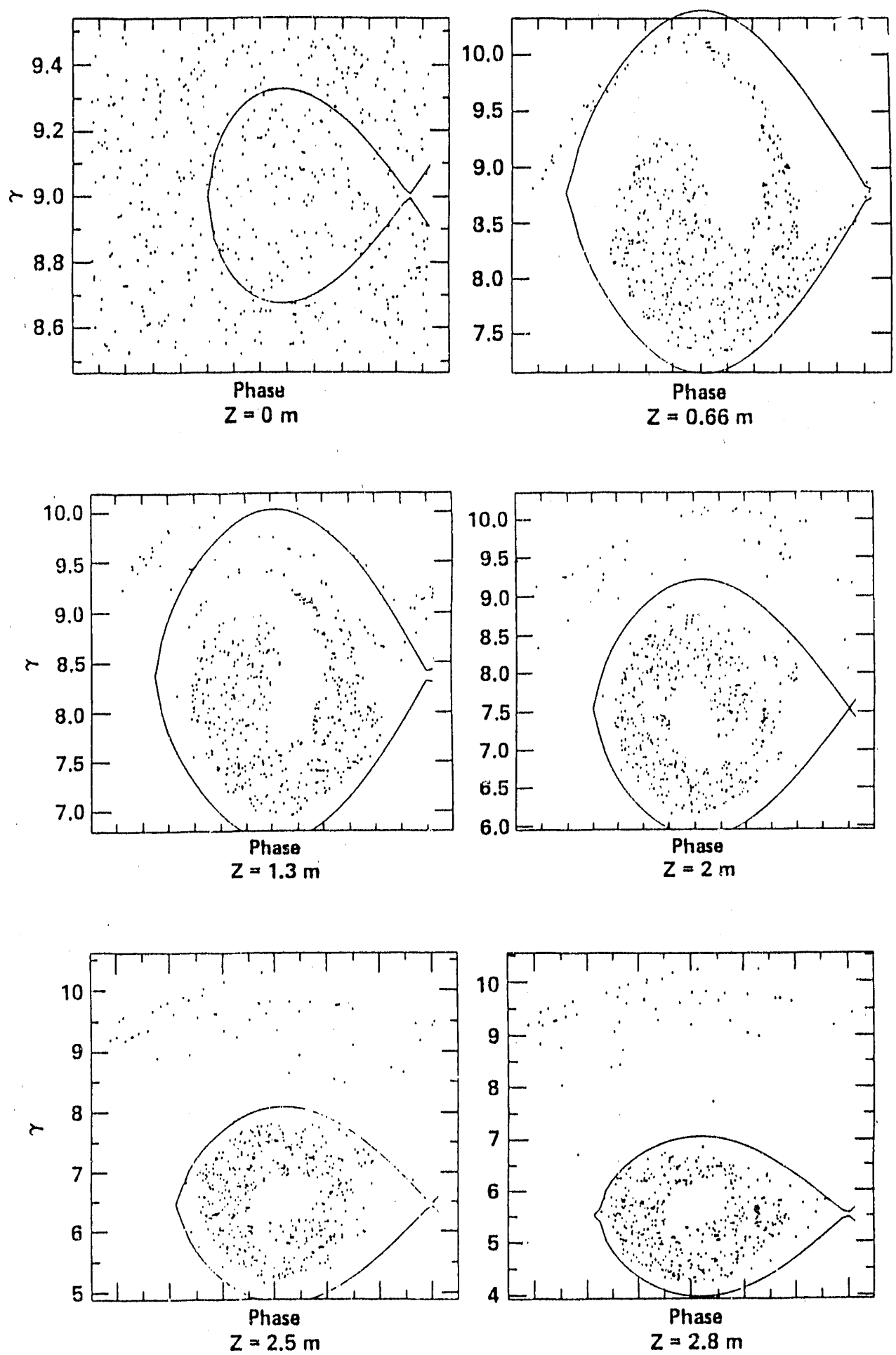

Fig. 4. Particle simulation (1D) showing capture and extraction in a particular FEL. The graphs are of relativistic factor $\gamma\left(\right.$ energy $\left.=\mathrm{mc}^{2}\right)$ vs. phase at various positions in the wiggler. Initially the particles have all phases and a $10 \%$ spread in energy. The parameters are $\left.I=3 \mathrm{kA}, \mathrm{E}=4 \mathrm{MeV}, \lambda_{\mathrm{w}}=10 \mathrm{c} n, \mathrm{~B}_{\mathrm{w}}(\mathrm{z}=0)=5 \mathrm{kG}\right)$. 


\section{III.8 Optical Guiding}

In the analysis described in the last Section it was assumed that the signal wave was amplified and phase shifted, but otherwise unaffected, by the free electron laser (FEL). This assumption is not correct, for the FEL affects both the amplitude and phase of the signal wave, and hence its transverse structure.

A simple way to describe the effect is to introduce a complex index of refraction, $n$, such that

$$
\begin{aligned}
& R_{\mathrm{e}}(\mathrm{n})-1=\frac{1}{\mathrm{k}} \frac{\mathrm{d} \varphi}{\mathrm{dz}}, \\
& I_{m}(n)=\frac{1}{\mathrm{ke}} \frac{\mathrm{de} s}{\mathrm{dz}},
\end{aligned}
$$

where a wave of amplitude, $E_{s}$ and wave number $k$, is characterized by its phase $\varphi$, and its normalized amplitude $\mathrm{e}_{\mathrm{s}}$, where

$$
\mathrm{e}_{s}=\frac{\mathrm{eE} s}{\sqrt{2} \mathrm{mc}^{2}} .
$$

It is then simple to derive a formula for the index of refraction created by interaction of the wave $E_{S}$ with the electron beam:

$$
\begin{gathered}
\mathrm{R}_{\mathrm{c}}(\mathrm{n})-1=\frac{2 \pi \mathrm{ea}_{\mathrm{w}}}{\mathrm{mc}^{3} \mathrm{ke}_{s}}\left\langle\frac{\cos \psi}{\gamma}\right\rangle, \\
\mathrm{I}_{\mathrm{m}}(\mathrm{n})=\frac{2 \pi \mathrm{eJa}_{\mathrm{w}}}{\mathrm{mc}^{3} \mathrm{ke}_{s}}\left\langle\frac{\sin \psi}{\gamma}\right\rangle,
\end{gathered}
$$

where $J$ is the beam current density and $\psi$ is the phase of a beam electron in the ponderomotive well of the FEL. 19

Because the electron beam is concentrated along the axis of the FEL, the light beam will experience the above index of refraction only within the electron beam (namely, along the axis), but not at other locations. Since an FEL causes the phase of 
the light beam to ever increase, the interaction produces focusing of the light beam ${ }^{19}$ as shown in Fig. 5.

Careful analysis of this highly nonlinear phenomena has been given by a number of workers. ${ }^{20,21}$ It is very important, in practice, for the high-power operation of an FEL.

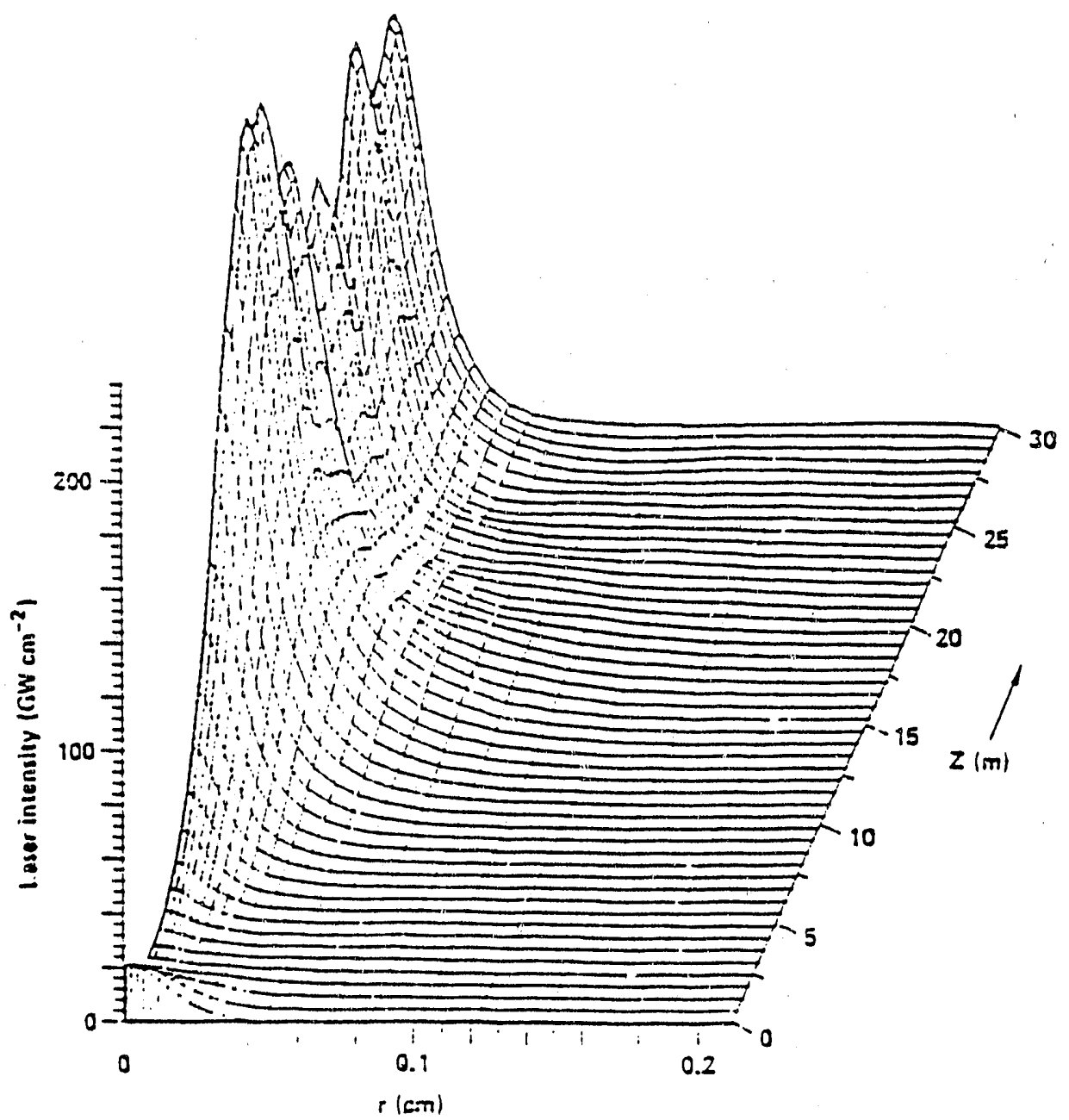

Fig. 5. Optical guiding in an FEL for $I=270 \mathrm{~A}, E=1 \mathrm{GeV}, \lambda_{\mathrm{s}}=2500 \AA$, $\lambda_{\mathrm{w}}=10 \mathrm{~cm}$. 


\section{III.9 Frequency Up-Shifting}

An electromagnetic wave (frequency $\omega_{0} / 2 \pi$ ) traveling through a medium which is slowly ionized must always satisfy the dispersion relation

$$
\omega^{2}=\omega_{p}^{2}+k^{2} c^{2}
$$

where $\omega_{\mathrm{p}}$ is the plasma frequency, and $\mathrm{k}$ is the wave number of the radiation. If the medium changes slowly, and uniformly, then the wavelength of the light will not change, but its frequency will change. 22 Thus we obtain:

$$
\omega^{2}=\omega_{\mathrm{p}}^{2}+\omega_{0}^{2} \text {. }
$$

In this manner rather large up-shifting can occur for it is relatively easy to make the final plasma frequency considerably larger than the initial electromagnetic wave , frequency.

A much more complicated situation, and a much more interesting situation, occurs when the ionization is not spacially uniform, but there is a moving ionization front. 23 Analysis of this situation has been presented by a number of authors. 24,25 The frequency up-shift depends upon whether the front is overdense or underdense in its own frame. If it is overdense the shift is $4 \gamma^{2}$, while if it is underdense the shift is

$$
\left(1+(1 / 4) \frac{\omega_{p}^{2}}{\omega_{0}^{2}}\right) \text {. }
$$

The rather complicated, and nonlinear, problem has been formulated in Ref. 24 . Even here, however, the assumption is made that the ionization front has a motion, and spacial description, which is independent of the electromagnetic wave. For the very powerful lasers which can be built these days, tha is not true; i.e., the laser pulse ionizes the plasma as it moves through it, and its rate of propagation is therefore determined by the wave amplitude. Study of this highly nonlinear problem, and determination of the frequency up-shifting one can so obtain, has been done with numerical simulation. ${ }^{23}$ This work could profit from more analytic studies. 
IV. Conclusions

It has been seen, by surveying a range of phenomena, that understanding of nonlinear motion is essential to the manipulation of particle beams. With ever-increased understanding of these nonlinear effects we have been able to design, build, and operate ever-more sophisticated devices. I have every reason to believe that this will be true in the future just as it has been so in the past.

References

1. Month, M. and Herrera, J., editors, Nonlinear Dynamics and the BeamBeam Interaction, AIP Conference Proceedings 57, New York (1979).

2. Koop, I. and Tumaikin, G., editors, Third Advanced ICFA Beam Dynamics Workshop, Siberian Division of the USSR Academy of Sciences, Novosibirsk (1989).

3. Visnjic, V." "Study of Dynamic Aperture of Low Beta Lattices at Tevatron Collider", Fermilab-Pub-90/80 (1990) (unpublished), submitted to Phys. Rev. D.

4. Symon, K.R. and Sessler, A.M., "Methods of Radio Frequency Acceleration in Fixed Field Accelerators", Proceedings of the CERN Symposium 1, 44 (1956).

5. Cole, F.T., "Longitudinal Motion in Circular Accelerators", in Physics of Particle Accelerators, editors Month, M. and Dienes, M., American Institute of Physics Conference Proceedings 153, 44 (1987).

6. Courant, E.D., "Beam Instabilities in Circular Accelerators", IEEE Trans. on Nucl. Science NS-12, 550 (1965).

7. Month, M., "Nature of the Beam-Beam Limit in Storage Rings", IEEE Trans. on Nucl. Science NS-22, 1376 (1975).

8. Keil, E., "Beam-Beam Effects in Electron and Proton Colliders", Proceedings of the IEEE Particle Accelerator Conference 89CH2669-0, editors Bennett, F. and Kopta, J., 1731 (1989). 
9. Bane, K.L.F., "Wakefield Effects in a Linear Collider", in Physics of Particle Accelerators, editors Month, M. and Dienes, M., American Institute of Physics Col ierence Proceedings, AIP Conference Proceedings 153, 971 (1987).

10. Whittum, D.H., Sessler, A.M. and Neil, V.K.,"Transverse Resistive Wall Instability in the Two-Beam Accelerator", Lawrence Berke ley Laboratory LBL-29125 (unpublished), to be published in Phys. Rev. A (1990).

11. Balakin, V.E., Novokhatsky, A.V. and Smirnov, V.P. "VLEPP:

Transverse Beam Dynamics", Proceedings of the 12th Internat. Conf. on High-Energy Accelerators, editors Cole, F.T. and Donaldson, R., Fermi National Accel. Lab., Batavia 119 (1984).

12. Hollebeek, R., Nucl. Instr. and Meth. 184, 333 (1981).

13. Yokoya, K., Nucl. Instr. and Meth. A251, 1 (1986).

14. Chen, P. Part Accelerators 20, 171 (1987).

15. Chen, P., et al, Phys. Rev. Lett. 64, 1231 (1990).

16. Whittum, D.H., Sessler, A.M. and Dawson, J.M., Phys. Rev. Lett. 64, $2511(1990)$.

17. Kroll, N.M., Morton, P.L., and Rosenbluth, N.M., IEEE Journ. Quantum Electronics QE-17, 1436 (1981).

18. Bonifacio, R., De Salvo Souza, L., and Pellegrini, C., editors High Gain, High Power Free Electron Lasers, North Holland, Amsterdam (1989).

19. Scharlemann, E.T., Sessler, A.M. and Wurtele, J.S., Phys. Rev. Lett. 54, 1925 (1985).

20. Moore, G.T., Opt. Comm. 52, 46 (1984); 54, 121 (1985).

21. Xie, M., Deacon, D.A.G. and Madey, J.M.J., "Eigenmode Analysis of Optical Guiding in Free Electron Lasers", Lawrence Berkeley Laboratory LBL-26987 (1989) (unpublished), submitted to Phys. Rev. A. 
22. Wilks, S.C., Dawson, J.M., and Mori, W.B., Phys. Rev. Lett. 61, 337 (1988).

23. Wilks, S.C., et al, Phys. Rev. Lett. 62, 2600 (1989).

24. Esarey, E., Ting, A., Sprangle, P., "Frequency Shifts Induced in Laser Pulses by Plasma Variations", Naval Research Laboratory Memorandum 6541 (1989) (unpublished).

25. Mori, W.B., "The Generation of Tunable Radiation Using A Relativistic Ionization Front" (1990), submitted to Phys Rev Lett. 

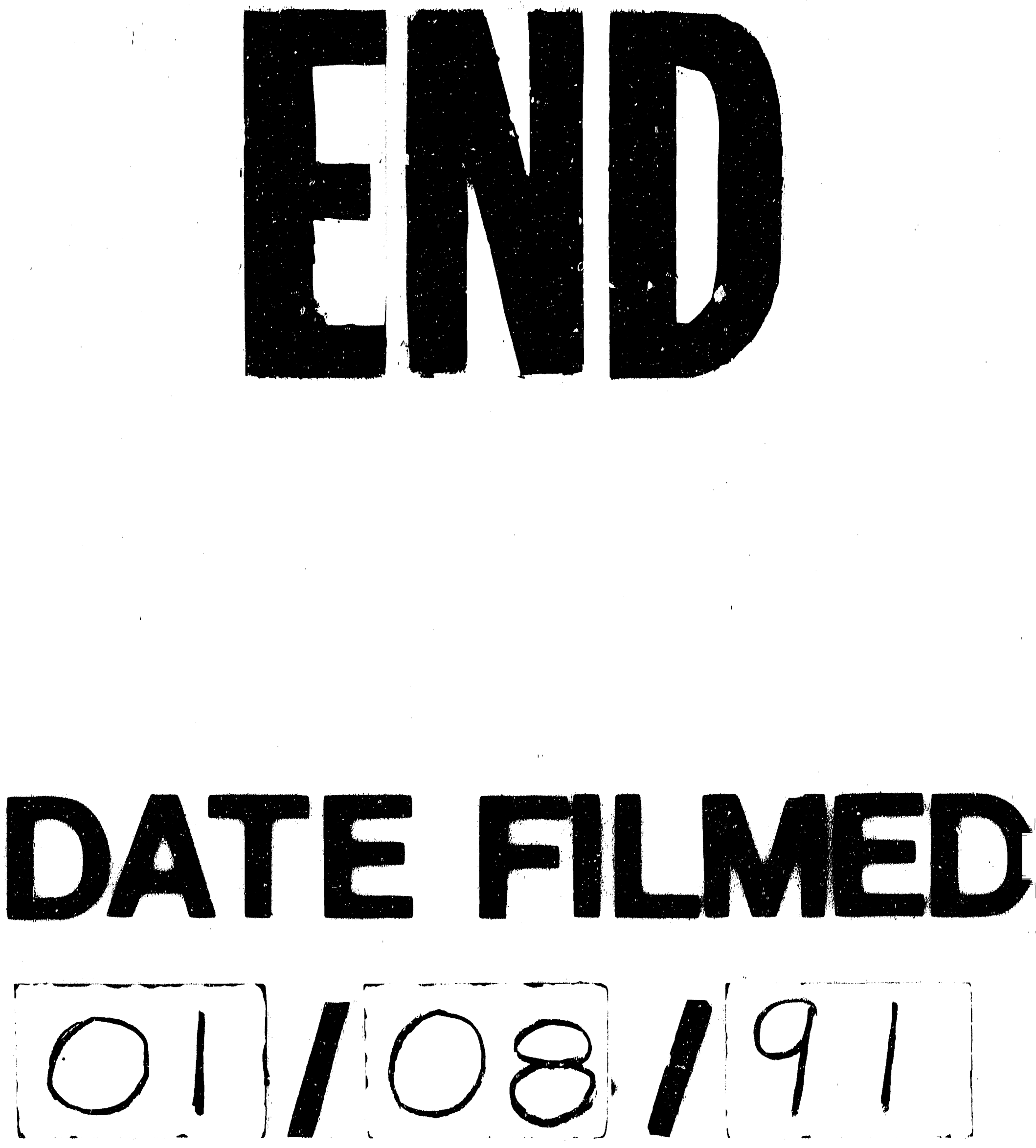
